\title{
Beitrag zur
}

\section{Kenntnis der Süsswasserfauna von Celebes.}

\section{ENTOMOSTRACA}

\author{
von \\ Dr Theodor STINGELIN. \\ Olten (Schweiz.)
}

Hierzu Tafel 14.

Die wenigen hier beschriebenen Entomostraken stammen aus einem Teiche der Umgebung von Makassar (Celebes). Sie wurden dasselbst im Jahre 1895 von den Celebesforschern $D^{r}$ Paul und $\mathrm{D}^{\mathrm{r}}$ Fritz SARAsIN gesammelt und bei ihrer Rückkehr nach Basel mir zur Bearbeitung übergeben. Die Durchsicht des Materials ergab folgenden Tierbestand:

1. Copepoden: 3 Arten in wenigen erwachsenen Exemplaren, aber vielen Larven.

2. Cladoceren: 3 Arten (wenige Individuen).

3. Ostracoden: 2 Arten in vielen erwachsenen Exemplaren und zahlreichen Larven.

Ausserdem unbestimmbar: Zwei Insektenlarven, eine Hydrachnidenlarve und ein zerfallener Panzer eines Rädertieres.

Aus dieser Zusammensetzung des Materials und dem Ueberwiegen plumper, schwerfälliger Ostracoden, geht hervor, dass dieser Fang dem Strande eines seichten, schlammigen Süsswasserteiches entnommen wurde. 
Bei der heute noch-so lückenhaften Kenntnis microscopischer Lebewesen aus Süsswasserbecken der Sundainseln lohnt es sich, auch das spärlichste von dort stammende Material einer eingehenden Prüfung zu unterziehen, und so hat, obschon arm an Arten, auch dieser kleine Fang wieder mehrere interessante Gesichtspunkte zu Tage gefördert.

Richard und Moniez haben im Jahre 1891 eine Anzahl Entomostraken von Celebes und Sumatra beschrieben (20) ${ }^{1}$. Wie RichaRD, so fand auch ich wieder:

\section{Cyclops leuckarti Claus ${ }^{2}$.}

Eine Copepodenspecies von weitester Verbreitung, die fast überall, wo eingehend nach Copepoden geforscht wird, sich findet. Trotz ihrer kosmopolitischen Verbreitung variiert diese Art selten und dann nur unbeträchtlich. Meine Exemplare stimmen mit SchveIL's Beschreibung (22) völlig überein. Eine Differenz besteht einzig in der Grösse. Die Länge meiner Individuen beträgt im Mittel 1,5 mm.

Ferner beschreibt RichaRd eine neue Cladocerenspecies des Genus Diaphanosoma (Daphnella) aus Sumatra. In seiner Revision des Cladocères 1895 (15) nennt er sie Diaphanosoma sarsi Richard. Eine Varietät dieser Art fand sich in meinem Material. Ich nenne sie:

2. Diaphanosoma sarsi Richard var. nova celebensis mihi (Fig. 1 und 2).

Sie variiert in folgenden Punktén:

Grösse: Sommereierweibchen messen 0,88-0,9 mm. in der Länge und sind $0,42-0,5 \mathrm{~mm}$. hoch. Diese Varietät ist eine

${ }^{1}$ Die in Klammer gesetzten Zahlen weisen auf die entsprechende Nummer im Litteraturverzeichnis hin.

${ }^{2} \mathrm{Da}$ in vorliegender Arbeit nur wenige Entomostraken-Species zur Behandlung kommen, sehe ich von einer systematischen Reihenfolge ab und erwähne zuerst die schon früher bekannt gewordenen Formen. 
plumpere, weniger flinke, litorale Form. Die meisten Exemplare waren von Vorticellen und anderen Infusorien besetzt.

Der Kopf misst ${ }^{1}$ /3 der Körperlänge. Sein vorderer Teil, vom grossen linsenreichen Auge fast ganz ausgefüllt, ist gleichmässiger gerundet, als aus RichaRD's Zeichnung der Diaphanosoma sarsi zu ersehen ist. Der ventrale Kopfrand sowohl als der dorsale besitzt eine schwache Impression.

Schale: Die Schalenklappen sind nicht retikuliert. Ihre Matrix ist mit gruppenförmig angeordneten, dicht gedrängten Нӧckerchen besetzt. Eine wesentliche Abweichung besteht in der Ausbildung des ventralen, hinteren Schalenteiles (Fig. $2 a$ und $b$ ). Die Schalenränder sind dort, ähnlich wie bei $D$. sarsi, eingeschlagen (Fig. $2 b$ ). Sie besitzen gleichfalls einen rundlichen, lappenartigen Vorsprung (Fig. 2c), und zu beiden Seiten desselben eine Einbuchtung ${ }^{1}$. Von der inneren aus (Fig. $2 d$ ), zieht sich bei RichaRD's Form eine ununterbrochene Reihe starker Borsten über den ganzen Hinterrand der Schale hinweg, während bei meiner Varietät diese Art der Bewehrung nicht ausgeprägt ist. Erst gegen die Mitte des hinteren Schalenrandes macht sich eine Flur feinster, mit stärksten Objektiven bloss erkennbarer Börstchen (Fig. $2 e$ ) bemerkbar, welche dorsalwärts eher kürzer als länger werden und oberhalb des Schalenrandes inseriert sind. Der lappenartige Vorsprung (c) trägt feine, lange und weit von einander abstehende Haare (Fig $2 f$ ).

Die Ruderantennen erreichen den hinteren Schalenrand nicht. Ihr kräftiger Stamm ist kürzer als der dorsale Schwimmast. Ob das Postabdomen mit demjenigen der D. sarsi übereinstimme, geht aus RichaRD's Beschreibung und Zeichnung nicht mit Sicherheit hervor. Auf die Darstellung dieses für die Cladocerenbestimmung so wichtigen Merkmales sollte immer die grösste Sorgfalt gelegt werden. Indem ich in Fig. 1 eine genaue Nach-

\footnotetext{
${ }^{1}$ Fig. 2 a zeigt diesen Schalenrand ausgebreitet. Längs der Linie $x-y$ ist er in Wirklichkeit eingeschlagen, so, wie durch Fig. $2 b$ dargestellt wird.
} 
bildung dieser Extremität gebe, sehe ich von einer längeren, umständlichen Beschreibung ab. Es sei nur noch bemerkt, dass die drei Dornen (Fig. . 1 b) sehr schlank, ungleich lang und wenig divergierend sind. Die Seiten des Postabdomens sind dicht und unregelmässig behaart. Die Postabdominal-Schwimmborsten (Fig. 1 c) sind $0,55 \mathrm{~mm}$. lang. Ihr äusseres Glied ist bloss halb so dick als dass innere. Das Auffinden einer litoralen Varietät . der pelagischen $D$. sarsi Richard bestätigt von neuem die Vermutung RicHARD's, dass die besprochene Art eine im hinterindischen Archipel weit verbreitete und ihm eigene sei.

3. Moina paradoxa Weismann (Fig. 3).

Aus Sumatra beschrieb RICHARD auch Moina weberi, ebenfalls eine hyaline Form mit rein pelagischen Charakteren. Meine Moinen aus Celebes scheinen im Gegensatz dazu wieder ächte Sumpfbewohner zu sein. Gelblich' gefärbt und plump, sind sie im Mittel $1 \mathrm{~mm}$. lang und $0,5 \mathrm{~mm}$. hoch. Die Sommereierweibchen sind stark kugelig aufgetrieben durch die grosse Zahl der Embryonen im Brutraume. Der Kopf ist 0,33-0,35 mm. lang, gedrungen und ohne dorsale Einbuchtung über dem Auge. Die Riechantennen (0,26 mm. lang), sowie die Ruderantennen, sind behaart, jedoch nicht so kräftig wie bei Moina paradoxa anderer Autoren. Das Postabdomen besitzt ebenfalls einen langen, schmalen Verschlussfortsatz für den Brutraum. (Diesem Daphnoiden-Merkmal lege ich, mit G. BuRcknaRDT (4), grössere systematische Bedeutung bei). Die Analränder sind mit neun bewimperten Zähnen bewehrt. An den Endkrallen sitzt ein aus ziemlich langen und feinen Borsten zusammengesetzter Nebenkamm. Die Schalenoberfläche lässt bisweilen undeutlich anostomosierende Linien erkennen. Bewehrung des Schalenrandes siehe Fig. 3.

Aus der Durchsicht der Moinenlitteratur geht hervor, dass obige Form als schwach ausgeprägte Varietät der Moina paradoxa an- 
zusehen ist. Mit Ausnahme davon, dass die Behaarung der Riechund Ruderantennen nicht so kräftig ist, wie bei der Stammform, und dass die Borsten des Nebenkammes an der Endkralle des Postabdomens feiner und länger sind, konnten keine wesentlichen Unterschiede herausgefunden werden, die zur Aufstellung einer besonderen Varietät obiger Art berechtigen würden. Sehr zu bedauern ist, dass sich keine Ephippienweibchen vorfanden.

\section{Alona sarasinorum nov. spec. mihi (Fig. 4 und 5).}

Dieser Lynceïde tritt in seinem ganzen Habitus der Alona intermedia Sars am nächsten. Er ist jedoch grösser als die weit verbreitete SARS'sche Art. Seine Länge beträgt 0,46-0,52 mm., die Höhe 0,36-0,38 mm. Ausserdem zeigten sich bei eingehender Betrachtung eine Anzahl wesentlicher Verschiedenheiten, welche mich bestimmten eine neue Art aufzustellen ${ }^{1}$.

Äussere Körperformen : Der dorsale Schalenrand (Fig. 4a) ist von vorne bis hinten gleichmässig gewölbt. Die ventralen Schalenränder sind schwach convex und nicht wellig gebogen. Sie tragen einen gleichmässigen Besatz mittelgrosser Borsten. Innerhalb des ganzen Hinterrandes verläuft ein Saum feinster Härchen. Die Schale ist structurlos, nur bisweilen erscheint sie fein punktiert. Der Kopf (Fig. 4) läuft in einen breiten, abgestumpften Schnabel (b) aus, der die gleiche Länge hat wie die Tastantennen (c). Der viereckige Augenfleck (d) ist grösser als das Auge (e). Der Lippenanhang ( $f$ ) ist gleichmässig gerundet und wird von den Armen der Ruderantennen $(g)$ nicht überragt. Das

${ }^{1}$ So sehr ich sonst der voreiligen Artenmacherei abhold bin, so finde ich doch darin das einzige Mittel, eine Tierform vor späterer Nichtbeachtung zu sichern. Denn selbst mit keiner der vielen von RICHARD geschaffenen aussereuropäischen Arten konnte ich die vorliegende Alona identificieren. In der Bewehrung des Postabdomens besteht einzig etwelche Ähnlichkeit mit Alona sarsi, einer von RichaRd schon aus Luwu (Celebes) beschriebenen Art (20). Es bleibt einer monographischen Bearbeitung der Alonen vorbehalten die zahlreichen Formen zu gruppieren und zu reducieren. Vorläufig ist es Pflicht der Specialforscher, die vielen Ausbildungsformen genau zu beschreiben und zu zeichnen. 
Postabdomen (Fig. 5) ist vorne spatelförmig verbreitert. Der postanale Höcker (a) ist schwach entwickelt und weit nach hinten gerückt. In dem davor liegenden breiten Ausschnitte (b) befindet sich die Analöffnung. Die Postabdominalränder (c) scheinen bei schwächerer Vergrösserung dicht mit unregelmässig angeordneten Dornen besetzt; stärker vergrössert lassen sich Zähne erkennen, welche vorne zu zweien, weiter hinten zu dreien (d) beisammen stehen und die, allmählig kleiner werdend, sich über die Analränder hinüberziehen. Ueber dieser Zahnreihe stehen seitlich an den Postabdominalrändern acht Borstenkämme (fig. 5 e). Die Endkrallen (f), mit starkem Nebendorn $(g)$ versehen, lassen nur mit starkem Objektiv eine Strichelung erkennen. Uebrigens ist die Zahnbewehrung des Postabdomens bezüglich der Zahl und Anordnung ihrer Elemente bei verschiedenen Individuen ziemlich verschieden, so dass auf dieses Merkmal nicht allzu grosses Gewicht gelegt werden darf. Viel wichtiger scheint mir für die Gruppierung der Alona-Arten die äussere Form des Postabdomens, sowie die Lage und Grösse des Postanal-Höckers und der Analspalte zu sein.

\section{Canthocamptus spec.?}

Ein sehr zerfallenes Exemplar eines Harpacticiden aus dem Genus Canthocamptus konnte leider nicht bestimmt oder beschrieben werden. Eiersack, Antennen und Furcalborsten fehlten vollständig. Die Länge betrug 0,6 mm. Der aüsseren Form und Gliederung des Thorax, sowie einem einigermassen erhaltenen fünften Fusse nach zu schliessen, gehört die Art in die Nähe des Canthocamptus trispinosus Brady oder des Canthocamptus northumbricus Brady.

Im Sarasin'schen Material aus Celebes befanden sich ferner in grosser Menge zwei Ostracoden-Species. Dieser Fund ist umso erfreulicher, als beides Arten sind, welche ähnlich dem eingangs erwähnten Cyclops leuckartiClaus, weiteste kosmopolitische Ver- 
breitung haben und welche bisher nicht im hinterindischen Archipel gefunden wurden. Es sind dies: 1. Cypria ophthalmica Jurine und 2. Cypris virens Jurine; var. monilifera Brady. Merkwürdig ist es, dass keine dieser zwei Arten unter den sechs von Moniez im Weber'schen Material aus Luwu (Celebes) (20) gefundenen, noch unter den zwölf von BRADY aus Ceylon (1) beschriebenen Arten figurieren.

\section{Cypria ophthalmica Jurine}

ist ein guter Schwimmer und nebst Cypris virens Jurine die gemeinste und wohl am eingehendsten studierte Ostracodenart (5).

Die asiatische Form stimmt, die Grösse ausgenommen, im ganzen Bau mit der europäischen überein. Sie ist kleiner und weist die gleichen Maasse auf wie die von HerRIK und Turner beobachtete amerikanische Form (9). Die Weibchen sind $0,55^{\mathrm{mm}}$ lang und $0,36^{\mathrm{mm}}$ hoch. (VAVRa (26) giebt für die böhmischen Formen 0,7 mm Länge und 0,5 mm Höhe an). Die Männchen, die in meinem Material ebenso häufig wie die Weibchen vertreten waren, maassen nur $0,47 \mathrm{~mm}$ in der Länge und $0,27 \mathrm{~mm}$ in der Höhe.

7. Cypris virens Jurine var. monilifera Brady (1868).

Cypris virens ist eine allgemein verbreitete, zähe und grosse Tümpelform, die sich bloss parthenogenetisch fortpflanzt und nach Normany und Brady (2) nie in grösseren Gewässern zu finden ist. Auch im Brackwasser und sogar fossil hat man sie gefunden. Die von BRADY (2) aufgestellte Varietät monilifera unterscheidet sich von der Stammform dadurch, dass sich an ihren Hinterrändern eine Reihe von 8-10 starken Warzen (oder Höckerchen) befindet. Das ist alles, was BRADY über diese Abart, die er in Südafrika und in England gefunden hat und als subbrackische Varietät bezeichnet, angiebt. Mit ihr sind die aus Celebes stammenden Exemplare identisch. Sie sind jedoch nur 
1,37 $\mathrm{mm}$ lang und $0,77 \mathrm{~mm}$ hoch. Die Schalenumrisse und die Schalenbeborstung sind gleich wie bei Cypris virens Jurine. Auch der hyaline Saum am Vorderrand ist vorhanden. Lage und Form des Auges und der Muskeleindrücke und selbst die Furca stimmen mit VAVRA's (26) Angaben und Zeichnung überein. Different sind die Schwimmborsten des zweiten Antennenpaares bezüglich der Länge. Sie überragen die Endklauen.

8. Pseudodiaptomus poppei nov. spec. mihi (Fig. 6-10).

Das Genus Pseudodiaptomus wurde von HeRrik (Minnesota) im Jahre 1884 geschaffen. [Pseudodiaptomus pelagicus (9)]. Seither wurden noch von drei anderen Forschern verwandte Formen aufgefunden und mit folgenden Genusnamen belegt: Schmackeria : [POPPE und RICHARD 1890 (13.)] Heterocalanus : [Th. ScotT 1893 (7.)] und Weismanella: [F. DAHL 1894 (7.)]. Giesbrecht und SchneIL fassen in ihrer Zusammenstellung der Copepoda Gymnoplea (7.) alle neun beschriebenen Arten unter dem ältesten Genusnamen Pseudodiaptomus zusammen. Als zehnte Art soll hier eine Form aus Celebes beschrieben werden. Dieselbe ist der von Poppe und Richard (1890) beschriebenen Schmackeria forbesi (13) aus der Umgebung von Shanghai am nächsten verwandt und wie diese eine Süsswasserform. Es standen mir drei zum Teil beschädigte Weibchen und glücklicherweise noch ein Männchen zur Verfügung. Ihre Abdomina und fünften Schwimmfusspaare lassen auf den ersten Blick eine Verschiedenheit von den anderen bisher bekannten Arten erkennen ${ }^{1}$.

Beschreibung des Weibchens: (Fig. 6, 7 und 8).

\footnotetext{
${ }^{1}$ Nach Herrn S. A. Poppe in Vegesack, der mir in uneigennützigster Weise das letzte Exemplar seiner grundlegenden Arbeit über Schmackeria forbesi übersandte, nenne ich aus Dankbarkeit und Anerkennung die neue Art: Pseudodiaptomus poppei!
} 
Grösse: Ohne die Furcalborsten beträgt die Länge 1,2$1,36^{\mathrm{mm}}$, die Höhe 0,4-0,44 mm. Der Kopf und das erste Thoraxsegment sind vollständig zu einem Cep ha lo th or a x verschmolzen. Dieser ist viergliedrig. Seine beiden letzten Segmente sind auch mit einander verwachsen, jedoch so, dass ihre Grenze seitlich noch durch eine schwache Furche, an welcher beiderseits 6-7 Zähne stehen, angedeutet ist (Fig. 6 a). Der Hinterrand des zweiten und dritten Gliedes ist ebenfalls bedornt (Fig. 6 b). Das letzte Thoraxsegment läuft dorsal jederseits in einen spitzen Vorsprung aus (Fig. $6 c$ ). Der Hinterrand dieses Segmentes ist jedoch abgerundet und auf beiden Seiten noch mit je 6-7 Dornen bewehrt (d).

Das A bdomen ist, die Furca inbegriffen, fünfgliedrig. Die hinteren Ränder des ersten, zweiten und dritten Gliedes sind bedornt. Im übrigen weicht das Abdomen von demjenigen der Schmackeria forbesi in folgenden Punkten ab :

\section{Schmackeria forbesi :}

1. Glied : länger als die Furca.

2. " : kürzer als das dritte.

3. " : Gleich lang wie das Furcalglied.

4. Glied : halb so lang als die Furca. Furcalglieder 4 mal länger als breit.

\section{Pseudodiaptomus poppei:}

Gleich lang wie die Furca; an der Basis angeschwollen und beiderseits fein beborstet.

Nur wenig kürzer als das dritte.

$2 / 3$ so lang als das Furcalglied.

$1 / 3$ der Furcalänge.

Furcalglieder 6 mal so lang als breit. Innenrand mit spärlichen, feinen und langen Cilien besetzt.

Die Furcalborsten verhalten sich in Zahl, Länge und Form gleich wie bei Schmackeria forbesi.

Die I. Antenne ist 22 gliedrig und zeigt gleiche Grössenverhältnisse und Bewehrung ihrer Glieder wie Schmackeria forbesi. Am dritten Glied steht wiederum eine ausserordentlich lange und kräftige Borste. 
Die II. Antenne (Fig. 7) hat verschiedene Eigentümlichkeiten. Ihr äusserer Ast (b) ist vom Basalglied abgeschnürt, nicht aber gelenkig mit demselben verbunden. Der innere Ast (c) besteht aus drei Gliedern, wovon das kurze, schildförmige Basalglied (d) innen eine kurze Borste aufweist. Das zweite Glied ist aufgeblasen und trägt am Innenrande drei kürzere und distal zwei längere, stets zweigliedrige, Borsten. An der Basis des dritten und äussersten Gliedes sind ebenfalls zwei solche Borsten inseriert. Der Mitte dieses Gliedes fehlt die bei Schmackeria forbesi vorhandene Borste. Am Ende stehen wiederum drei sehr lange und stark bewimperte Schwimmborsten (Fig. 7 e). Im Bau der Mandibeln, Maxillen und Kieferfüsse konnte ich keine weiteren Abweichungen von Schmackeria forbesi erkennen. Auch das vierte Paar der Schwimmfüsse stimmt in allen Teilen mit POPPE und RichaRD's Fig. 9 völlig überein.

Im 5. Beinpa ar (Fig. 8), das noch etwelche Aehnlichkeit mit demjenigen der Schmackeria forbesi hat, treten schon stärkere Abweichungen auf. Auf den Basipodit (a) folgt auch ein zweigliedriger Exopodit (b), der in einen noch längeren, starken Stiletfortsatz (c) ausläuft. Das erste Drittel dieses Stilets weist keine seitliche Bewimperung auf. Die äussern zwei Drittel sind innen mit kräftigeren, aussen mit feineren Wimpern besetzt. Von der Basis des Stiletfortsatzes zweigt sich noch ein kürzerer, nach innen gerichteter Nebendorn (d) ab. Sodann geht vom zweiten Exopoditgliede ein breiter, klauenförmiger Fortsatz (e) aus. Derselbe ist beiderseits gleichmässig beborstet. Auf dem Aussenrand des zweiten Basipoditgliedes und der beiden Exopoditglieder steht schliesslich noch jeweilen ein kurzer Dorn. Am Innenrande des ersten Exopoditgliedes erhebt sich an Stelle des hyalinen Vorsprunges der Schmackeria forbesi ein breiter, dreieckiger, massiver Fortsatz (Fig. $8 f$ ). Borstenreihen mitten auf den Basalgliedern fehlen dem Pseudodiaptomus poppei. Leider trug keines der drei Weibchen einen Eiersack. 
Beschreibung des Männchens: (Fig. 9 und 10). Länge: $1,1^{\mathrm{mm}}$. Höhe: $0,35^{\mathrm{mm}}$. Das Abdomen setzt sich, die Furca inbegriffen, aus 6 Segmenten zusammen. Das erste Glied ist seitlich aufgeblasen und bewimpert. Zweites, drittes und viertes Glied ungefähr gleich lang, sind hinten jeweilen mit einem Dornenkranz ausgerüstet. Das fünfte Glied ist das kürzeste. Die Furca, sowie ihre Glieder und Anhänge, sind gleich beschaffen wie diejenigen des Weibchens.

Die I. Antenne ist 20 gliederig und stimmt in allen Teilen mit der von Schmackeria forbesi beschriebenen und gezeichneten überein, weshalb ich auf jene Beschreibung und Zeichnung verweisen kann.

5. Beinpaar: Am meisten weicht in seiner Ausbildung das 5. Schwimmfusspaar (links und rechts verschieden) von den entsprechenden Gliedern der männlichen Schmackeria forbesi ab. Es ist hier zu einem Prehensionsapparat von bizarrster Gestalt geworden.

Der fünfte Fuss rechts (Fig 9) ist viergliederig. Der Basipodit (a) ist conisch und mit kräftiger Muskulatur erfüllt. Der Entopodit fehlt gänzlich. Der Exopodit ist dreigliederig. Sein erstes Glied läuft distal in einen kräftigen Stiletfortsatz (b) aus, der bis zum Gelenk des dritten, äussersten Gliedes reicht. Das zweite Exopoditglied ist durch die sehr starken Bewegungsmuskeln des Endgliedes gewaltig aufgetrieben. Das dritte Glied (c), etwas länger als das vorige, ist schmal und eigenartig gekrümmt. Auf zwei vorgewölbten Stellen (Fig. $9 d$ und $e$ ) der Innenseite sitzt je ein kleiner Zahn. Die Endkralle ( $f$ ) ist bewimpert.

Der fünfte Fuss links (Fig. 10) gleicht eher einer fürchterlichen Waffe, als einem blossen Greiforgan. Der Basipodit (a) ist aussen mit zwei mörderischen Anhängen, einem pflugscharförmigen (b) und einem stiletförmigen (c) ausgerüstet. Der Entopodit ist zweigliederig (Fig. $10 d$ und e). Das innere Glied $(d)$ umschliesst die Bewegungsmuskeln des äusseren und ist dem ent- 
sprechend aufgetrieben. Am distalen, äusseren Ende, läuft es in einen breiten, scharf zugespitzten und bewimperten Fortsatz $\left(d_{1}\right)$ aus. Das äussere Glied (e) ist spatelförmig verbreitert und schnürt $\mathrm{zu}$ vorderst noch einen lappenförmigen, mit vier kurzen Dornen versehenen Vorsprung ( $f$ ) ab. - In der Mitte der Aussenseite des Endgliedes steht noch ein stark bewimperter, schlanker Dorn (g), der beinahe halb so lang ist als das äussere Glied. Diesem gegenüber sitzt schliesslich an der Innenseite des letzten Gliedes noch ein Büschel feinster Fäden ( $h$ ), welchen vielleicht eine Sinnesfunktion zukommt.

Die Durchsicht dieser wenigen, von den Herren SARAsIn gelegentlich in Celebes gesammelten niederen Crustaceen zeigt uns also, dass auch im hinterindischen Archipel die allgemein verbreiteten Entomostracen, wie Cyclops leuckarti Claus, Moina paradoxa Weismann und Cypria ophthalmica Jurine nur wenig oder gar nicht verändert zu finden sind. - Andere Formen sind bekannten europäischen Arten nahe verwandt, wie Alona sarasinorum nov. spec. mihi und der Canthocamptus spec. der C. trispinosus-Gruppe. Wieder andere sind den hinterindischen Inseln eigen, wie Diaphanosoma sarsi und ihre Varietät: Diaphanosoma sarsi Richard, nov. var. celebensis mihi. Und schliesslich stellen sich noch Formen ein, wie eine Cypris virens Jurine var. monilifera Brady und Pseudodiaptomus poppei, welche, wie erstere, schon im Brackwasser gefunden und dadurch marinen Formen sich annähern, oder die, wie letztere, ihre nächsten Verwandten fast ausnahmslos im Brackwasser, oder sogar im Meere haben und sich offenbar im Laufe der Zeit, wie Schmackeria forbesi und Pseudodiaptomus stuhlmanni Poppe uud Mrázek, dem Süsswasser angepasst haben ${ }^{1}$. Es ist wahrscheinlich, dass die eben erwähnten Formen auch noch im Brackwasser leben.

\footnotetext{
${ }^{1}$ Von den bisher bekannten Arten des Genus Pseudodiaptomus wurden sechs Arten im Brackwasser, eine ausschliesslich im Meere und zwei im Süsswasser gefunden.
} 
Die Herren $\mathrm{D}^{\mathrm{r}}$ Paul und $\mathrm{D}^{\mathrm{r}}$ Fritz SARAsin haben in ihrem Werke über Die Süsswassermollusken von Celebes (1898, Wiesbaden. KreideL's Verlag) einen Ausspruch von Martens zum Gesetze erhoben. Er heisst: «Die Aehnlichkeit der gesammten Süsswasserfauna mit der gesammten Meeresfauna nimmt zu von den Polen gegen den Aequator hin.

Für die Richtigkeit dieses Gesetzes sprechen also auch meine wenigen Befunde bei niederen Crustaceen.

Auffällig ist auch, dass die tropischen Süsswasserformen (wie aus den Beobachtungen anderer Forscher ebenfalls hervorgeht), allgemein, wenn auch mit europäischen Arten identisch, nicht, wie man etwa erwarten könnte, grösser und üppiger, sondern durchwegs kleiner sind. 


\section{Litteratur.}

1. Brady, G. S. Notes on Entomostraca, collected by H. A. Haly-Ceylon. Journal of Linnean soc. zoology, vol. 19. 1885.

2. Brady and Normann. Monograph of marine and freshwater Ostracoda. Scientif. Transactions of R. Dublin Soc. Serie II, vol. 4. 1889, et vol. ร. 1896.

3. Bradi and Robertson. Ostracoda of Tidal Rivers. Annals and magazine of nat. history. Serie 4, vol. VI. 1870.

4. Burckhardt, G. Faunistische und systematische Studien über das Zooplankton der Schweizerseen. Revue Suisse de Zoologie, Genève, T. 7. 1900.

5. Claus, C. Beiträge zur Kenntnis der Süsswasser-Ostracoden. Arbeiten des zool. Inst. Wien, vol. X. 1892.

6. Daday von, Eugen. Mikroscopische Süsswassertiere aus Ceylon. Anhangsheft zum XXI. Bande (1898) des Természetrajzi Füzetek. Budapest. 1898.

7. Giesbrecht und Schmeil. Crustacea Copepoda (I. Gymnoplea). Das Tierreich. 6. Lieferung. Berlin. Friedländer \& Sohn. 1898.

8. Hellich, B. Die Cladoceren Böhmens. Archiv der naturwiss. Landesdurchforschung von Böhmen. III. Bd.

9. Herrik, C. L., and Turner, C. H. Synopsis of the entomostraca of Minnesota. Second Report of the state zoologist, Zoological series II. S. Paul. 189.\%.

10. Martens. Ueber einige ostasiatische Süsswassertiere. Archiv für Naturgeschichte. 34. Jahrg., vol. I. 1868.

11. Мrazek. Schmackeria hessei. Sitzber. der k. böhm. Ges. der Wissensch. No 24. f. 1-3. Prag. 1894.

12. Pорев und Мrazeк. Schmackeria Stuhlmanni, Jahrbuch der Hamburger wiss. Anstalten, vol. 12, p. 125, f. I. 1894.

13. Poppe et Richard. Schmaclieria forbesi. Mémoires de la Soc. Zool. de France, vol. 3, tab. X. f. 1-14. 1890.

14. Richard, J. Entomostracés nouveaux ou peu connus. Bulletin de la Soc. Zool. de France, vol. XIII. 1888.

15. - Revision des Cladocères (Sidida et Holopedida). Annales des sciences naturelles, 7. série. Zool. 189.̈. Paris. 
16. Richard, J. Sur quelques Entomostracés d'eau douce d'Haïti. Mém. de la Soc. Zoolog. de France, tome VIII. 1895.

17. - Cladocères et Copepodes de Tiflis et du lac Golitsha. Bulletin de la Soc. Zoolog. de France, tome XX. 1895.

18. - Entomostracés de l'Amérique du Sud. Mém. de la Soc. Zool. de France, tome X. 1897.

19. - Sur quelques Entomostracés d'eau douce des environs de Buenos-Aires. Anales del museo nacional de B. Aires, tome V. 1897.

20. Richard et Moniez. Entomostracés d'eau douce de Sumatra et de Célèbes. in: Weber, Max. Zoolog. Ergebnisse einer Reise in Niederländ. Ost-Indien. Leiden 1891. Bd. II .

21. Sars, G. O. Australian Cladocera, (kurzes Referat über die Hauptarbeit), Journal of the microscop. society, 1887, ser. II. $7^{2}$.

22. Schmeit, Otto. Deutschlands freilebende Süsswasser-Copepoden. Bibliotheka Zoologica, vol. 21.

23. Scourfield, D. J. The Entomostraca of Epping forest. The Essex Naturalist, vol. X. 1898.

24. Steuer, Ad. Ein Beitrag zur Kenntnis der Cladoceren- und CopepodenFauna Kärntens. Verhandl. d k. k. zool.-bot. Ges. Wien, 1897.

25. Stingelin, Th. Die Cladoceren der Umgebung von Basel. Revue Suisse de Zool. Bd. III. Genf 189 כั.

26. Vavra, Wenzel. Monographie der Ostracoden Böhmens. Archiv der naturwiss. Landesdurchforschung Böhmens, vol. VIII. Prag 1891.

27. - Die von Dr F. Stuhlmann gesammelten Süsswasser-Ostracoden Zanzibar's. Beiheft zum Jahrbuch der Hamburger wissensch. Anstalten, XII. Bd. Hamburg 189 s.

28. - Die Süsswasser-Ostracoden Deutsch-Ostafrilias. Ostafrika IV. Berlin 1897. (Sep. Abzug.)

29. - Süsswasser-Ostracoden der Hamburger Magelhanischen Sammelreise. Hamburg, Verlag von Friedrichsen. 1898.

30. Weltner, W. Die Cladoceren Ost-Afrikas. in : Ostafrika IV. Berlin 1897. (Sep. Abzug.) 


\section{TAFEL 14}

\section{FIGURENERKL ERUNG}

(Die Detailerklärungen finden sich im Text eingeschaltet.)

Fig. 1. Postabdomen der weibl. Diaphanosoma sarsi Richard nov. var. celebensis mihi.

$2 a$ und $b$. Hinterer ventraler Schalenrand desselben Tieres.

3. Ventraler Schalenrand von Moina paradoxa Weismann aus Celebes.

4. Kopf mit Lippenanhang von Alona sarasinorum nov. spec. mihi.

ร. Postabdomen derselben Art.

6. Hinterer Teil des Cephalothorax von Pseudodiaptomus poppei nov. spec. mihi.

7. Zweite Antenne desselben Tieres.

8. Ein Fuss des fünften Schwimmfusspaares des weibl. P. poppei.

9. Rechter Fuss des fünften Beinpaares des männl. $P$. poppei.

10. Linker Fuss des fünften Beinpaares des männl. P. poppei. 


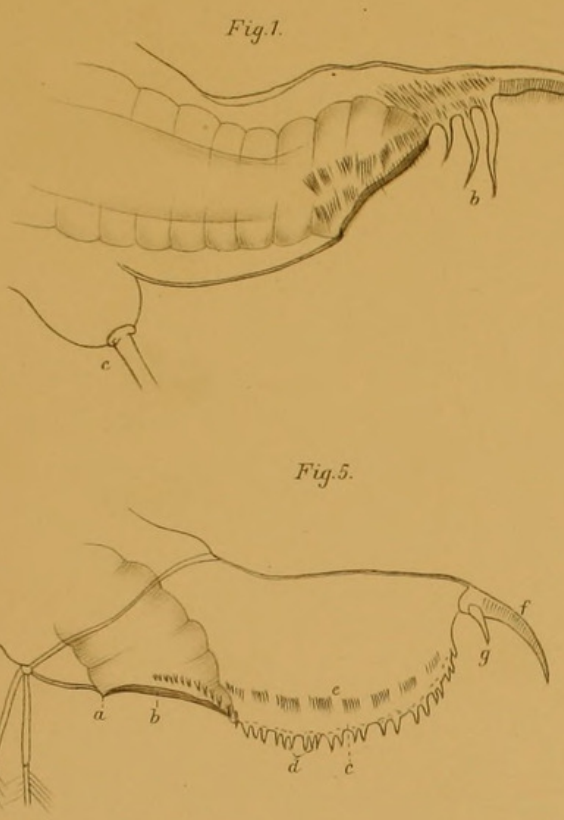

Fig. 7 .
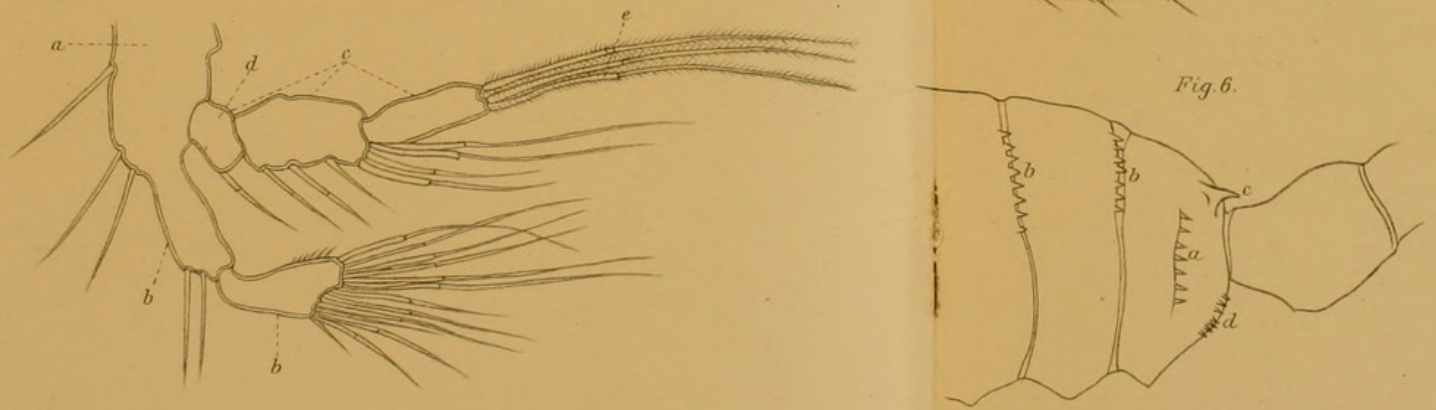

Fig. 10.
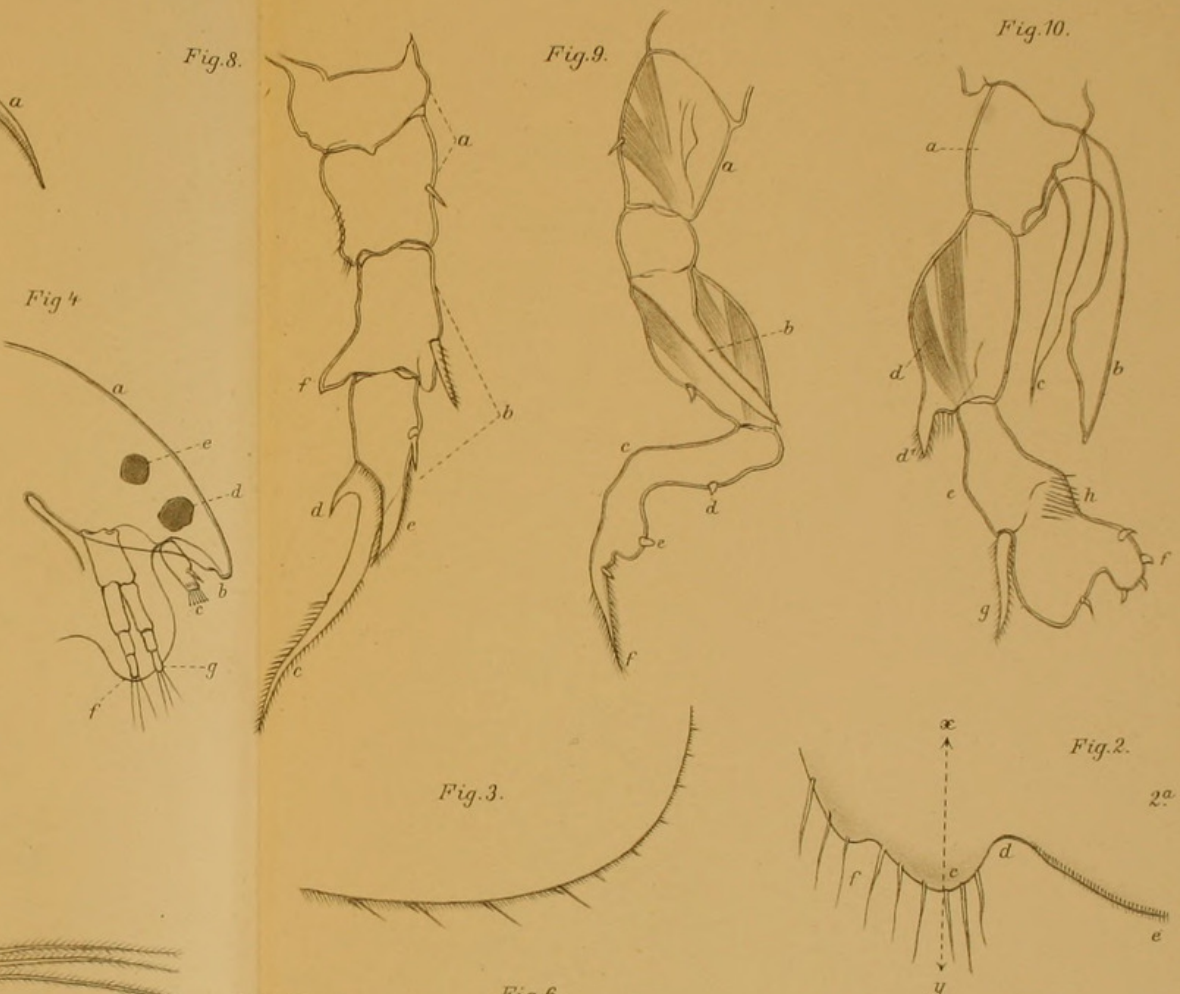

Th.Sfingelin del

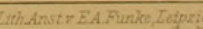

Th. Stingelin. Entomostraca. 


\section{$2 \mathrm{BHL}$ Biodiversity Heritage Library}

Stingelin, Theodor. 1900. "Beitrag zur Kenntnis der Süsswasserfauna von

Celebes. Entomostraca." Revue suisse de zoologie 8(2), 193-207. https://doi.org/10.5962/bhl.part.75156.

View This Item Online: https://www.biodiversitylibrary.org/item/38141

DOI: https://doi.org/10.5962/bhl.part.75156

Permalink: https://www.biodiversitylibrary.org/partpdf/75156

\section{Holding Institution}

MBLWHOI Library

\section{Sponsored by}

MBLWHOI Library

\section{Copyright \& Reuse}

Copyright Status: NOT_IN_COPYRIGHT

This document was created from content at the Biodiversity Heritage Library, the world's largest open access digital library for biodiversity literature and archives. Visit BHL at https://www.biodiversitylibrary.org. 